\title{
Beyond silicene: synthesis of germanene, stanene and plumbene
}

\author{
Junji Yuhara1* and Guy Le Lay2 \\ 1Graduate School of Engineering, Nagoya University, Nagoya 464-8603, Japan \\ 2Aix-Marseille Université, CNRS, PIIM UMR 7345, 13397 Marseille Cedex, France \\ *E-mail: j-yuhara@energy.nagoya-u.ac.jp
}

\begin{abstract}
Twenty five years after the first theoretical prediction of two-dimensional (2D) honeycomb-like silicon and germanium, now coined silicene and germanene, the last Group 14 artificial cousin of graphene has been synthesized, thus terminating the lineage from silicene (2012) to germanene (2014), stanene (2015), and finally plumbene (2019). Here, we describe the realizations and review the tantalizing properties of these outstanding novel 2D materials.
\end{abstract}

\section{Introduction}

The recent realization of epitaxial plumbene ${ }^{1}$ ) has ended the quest for the challenging synthesis of all Group 14 cousins of graphene with elemental monolayer honeycomb structure, as originally theoretically predicted. Indeed, ending this quest, does not mean halting research; instead, it will give a new impetus and launch many new studies on fundamental and applied aspects. At variance with graphene, these cousins have no parent crystal like graphite in nature, from which they could be possibly peeled off. Since the first realizations of silicene in $2012,{ }^{2-4)}$ showing that the initial theoretical predictions ${ }^{5,6)}$ could be effectively materialized, a gold rush has started to obtain elemental two-dimensional (2D) materials analogs, especially at present from element $\mathrm{B}$ in Group 13 to make borophene ${ }^{7)}$ to element $\mathrm{Bi}$ in Group 15 to make bismuthene. ${ }^{8)}$

A very recent article has comprehensively spanned all these novel 2D materials (except for plumbene, which had not yet appeared in reality at the moment of submission). ${ }^{9}$ ) Several books (see, e.g. Refs. 10 and 11), book chapters (see, e.g. Refs. 12 and 13), and review articles (see, e.g. Refs. 14-16) have been published, especially highlighting silicene, graphene's first cousin, whose realization has further engen- dered many offsprings and a huge number of theoretical and experimental studies. In this respect it is worth mentioning that the "growth and properties of silicene" has been ranked 4th topic (among 10 selected) in the Hot Research Fronts in physics, as determined following a Thomson-Reuters cita- tion-based study covering years 2011-2014. ${ }^{17}$ ) The next 10 Hot Research Fronts, issued in 2019, identifying the hottest and emerging specialty areas in scientific research in physics from 2013 to 2018 , have placed "2D Group-VA materials: antimonene, arsenene and bismuthene" also at the 4 th rank, a clear marker of the direct parentage. ${ }^{18)}$

Hence, we will rather focus, here, on our post-silicene achievements, obtained in recent years at Nagoya University in Japan, where specific growth methods have yielded a new arrangement of epitaxial germanene by segregation, ${ }^{19}$ ) as well as large-area planar stanene ${ }^{20)}$ and plumbene, ${ }^{1)}$ which developed above surface alloys initially formed. Germanene, stanene and plumbene, are especially exciting because they inherently possess large spin-orbit couplings (SOCs), which can make them robust topological insulators and may favor a Quantum Spin Hall effect near, or at RT and even above, which is highly promising for future devices and quantum information applications. 


\section{Germanene}

Already in 1994, 10 years before the advent of graphene, two pioneers, K. Takeda and K. Shiraishi, predicted from density functional theory (DFT) calculations the "Theoretical possibility of stage corrugation in Si and Ge analogs of graphite". 5) In other words, they demonstrated the possible existence of standalone atom-thin sheets of silicon and germanium, now coined silicene and germanene. Their article remained essen- tially unnoticed until 2009, when S. Cahangirov et al. resurrected it and confirmed the findings, further demon- strating the dynamical stability and underlining the appearance of Dirac fermions at the K and K' points of the surface Brillouin zone (SBZ). ${ }^{6}$ ) However, it was only after 2012, when silicene had been truly synthesized under ultra-high vacuum (UHV) conditions, either by Si deposition onto (111) surfaces of silver single crystals, 2,4$)$ the reversed process of growing silver on $\mathrm{Si}(111)$ to form the prototypical $\mathrm{Si}(111)(\sqrt{3} \times \sqrt{3})$-Ag surface reconstruction, $\left.{ }^{19}\right)$ or by segregation of silicon through a zirconium diboride thin film grown on a piece of silicon wafer, ${ }^{3)}$ that their citations started to rise quickly.

Two years later, germanene was synthesized in Marseille (France) by Ge deposition onto a gold (111) surface, $\left.{ }^{21}\right)$ again the reverse fashion of forming the $\operatorname{Ge}(111)(\sqrt{3} \times \sqrt{3})$-Au surface reconstruction. ${ }^{22)}$ This birth initiated the lineage down column 14 of novel elemental artificial 2D materials beyond graphene, from silicene to plumbene, as shown in Fig. 1. Compelling evidence of the synthesis of germanene was obtained, especially for a nearly flat phase forming large domains in scanning tunneling microscopy (STM) imaging. Advanced DFT calculations confirmed its atomic geometry, i.e. a $(\sqrt{3} \times \sqrt{3})$ germanene superstructure matching a $(\sqrt{7} \times \sqrt{7}) \mathrm{R} 19.1^{\circ} \mathrm{Au}(111)$ supercell. However, other $2 \mathrm{D}$ phases were simultaneously co-existing, making the situation somewhat complex.

\begin{tabular}{|c|c|c|}
\hline 13 & 14 & 15 \\
\hline${ }^{5} \mathrm{~B}$ & ${ }_{\text {graphene }}^{6}$ C & ${ }^{7} \mathbf{N}$ \\
\hline 13 & $\begin{array}{c}14 \\
\mathrm{Si}\end{array}$ & $\begin{array}{r}15 \\
P\end{array}$ \\
\hline$\stackrel{31}{\mathrm{Ga}}$ & $\begin{array}{l}32 \\
\mathrm{Ge}\end{array}$ & $\begin{array}{l}33 \\
\text { As }\end{array}$ \\
\hline $\begin{array}{l}49 \\
\text { In }\end{array}$ & $\begin{array}{l}50 \\
\text { Sn }\end{array}$ & $\begin{array}{l}51 \\
\mathrm{Sb}\end{array}$ \\
\hline $\begin{array}{c}81 \\
\text { TI }\end{array}$ & $\begin{array}{l}82 \\
\mathrm{~Pb}\end{array}$ & $\begin{array}{c}83 \\
\mathrm{Bi}\end{array}$ \\
\hline
\end{tabular}

Fig.1: The lineage of artificial Group 14 elemental novel 2D materials from silicene to plumbene. 
Instead, a highly desirable single phase of germanene, technically very important for the easy synthesis and transfer of this promising 2D electronic material, was recently prepared by a segregation procedure through a thin $\mathrm{Ag}$ (111) film epitaxially grown on a $\mathrm{Ge}(111)$ piece of wafer. This method, described in Fig. 2, is different from the molecular beam epitaxy (MBE) one used previously, but bears resemblance with the preparation of silicene by segregation through a $\mathrm{ZrB}_{2}$ thin film, as mentioned above.

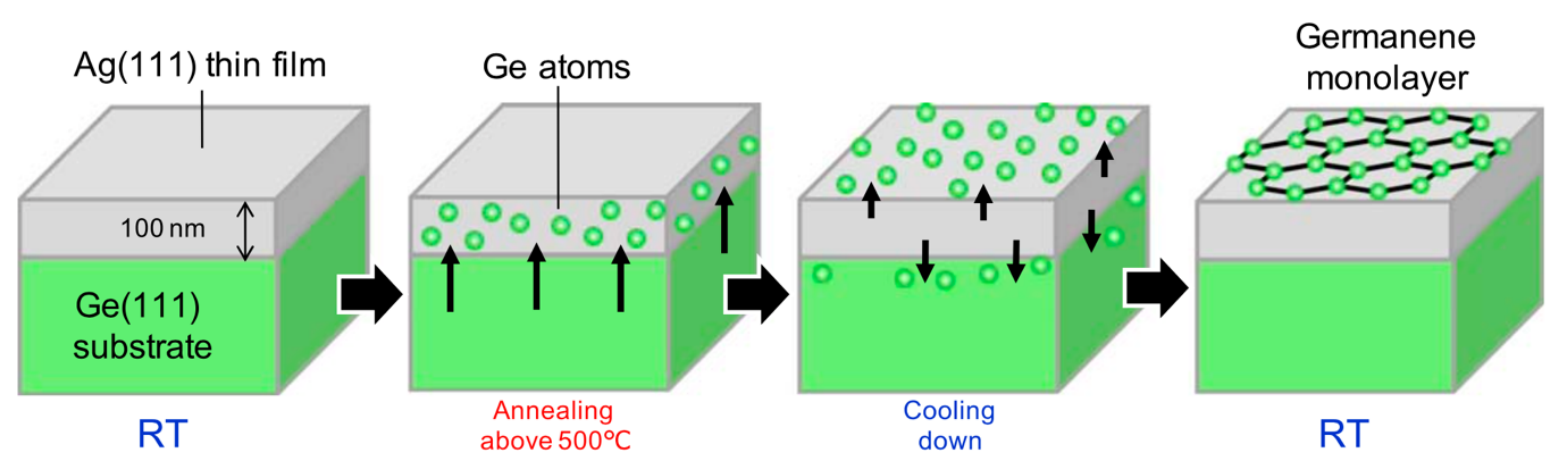

Fig. 2: Preparation of germanene by segregation through a silver (111) thin film.

From the STM images, which clearly reveal an internal honeycomb arrangement with low buckling within $0.01 \mathrm{~nm}$, the surface is completely covered with a self-limiting atom-thin layer showing a highly ordered long-range superstructure in wide scale. Two types of protrusions, named hexagon and line, form a $(7 \sqrt{ } 7 \times 7 \sqrt{ } 7) \mathrm{R} 19.1^{\circ}$ supercell with respect to $\mathrm{Ag}(111)$, with a very large periodicity of $5.35 \mathrm{~nm}$, which corresponds to a $(3 \sqrt{2} 1 \times 3 \sqrt{2} 1) \mathrm{R} 10.9^{\circ}$ germanene superstructure. Interestingly, this shows that the two preparation methods, either by deposition or by segregation are asymmetric, leading to different final situations. Figure 3 displays the sharp low energy electron diffraction (LEED) pattern obtained after segregation with incommensurate "( $1.35 \times 1.35)$ "R $30^{\circ}$ spots, corresponding to a lattice constant of $0.39 \mathrm{~nm}$, in good agreement with the theoretical lattice constant of freestanding germanene; it also shows a typical STM image and the derived atomic model.

As aluminum has essentially the same lattice parameter as gold and silver, germanene has been also prepared in 2015 by deposition onto $\mathrm{Al}(111)$ single crystal surfaces, where it appeared as a $(3 \times 3)$ reconstruction in LEED patterns. ${ }^{23)}$ However, other phases are also simultaneously formed. ${ }^{24)}$ A well-defined Dirac cone remained elusive but a clear metallic behavior was observed in initial scanning tunneling spectro- scopy measurements, which evolved in a diversity of spectra explained by the erratic modifications of the tip apex occurring during the scans. ${ }^{25)}$ 
(a)

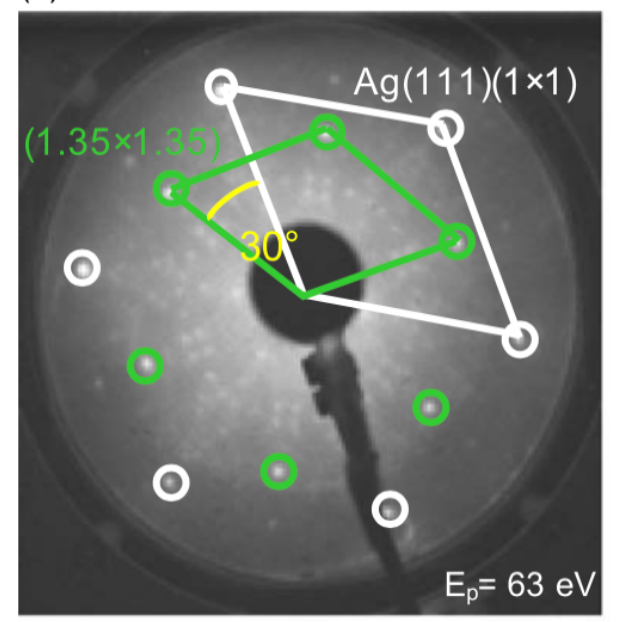

(b)

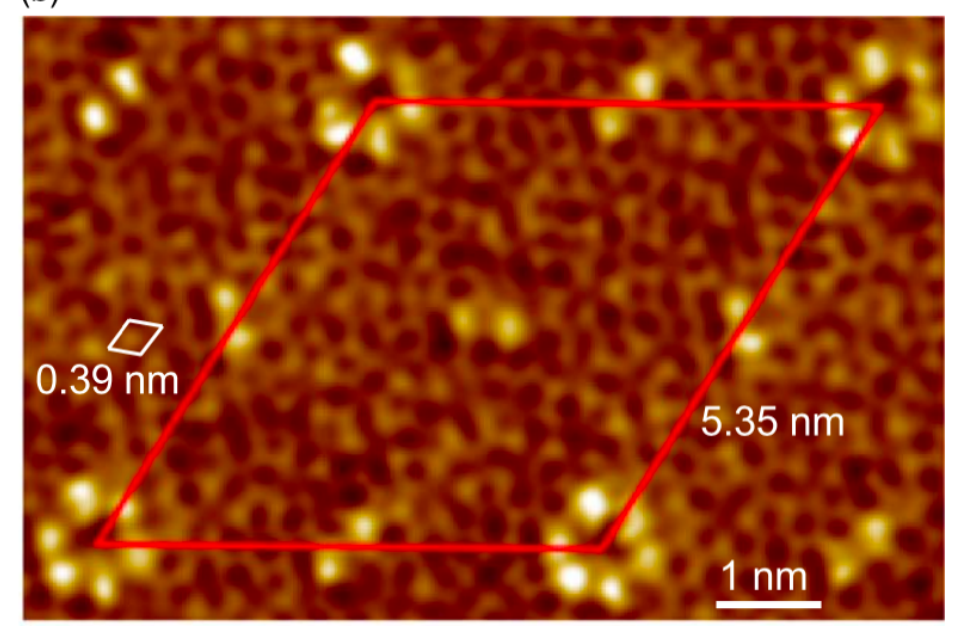

(c)

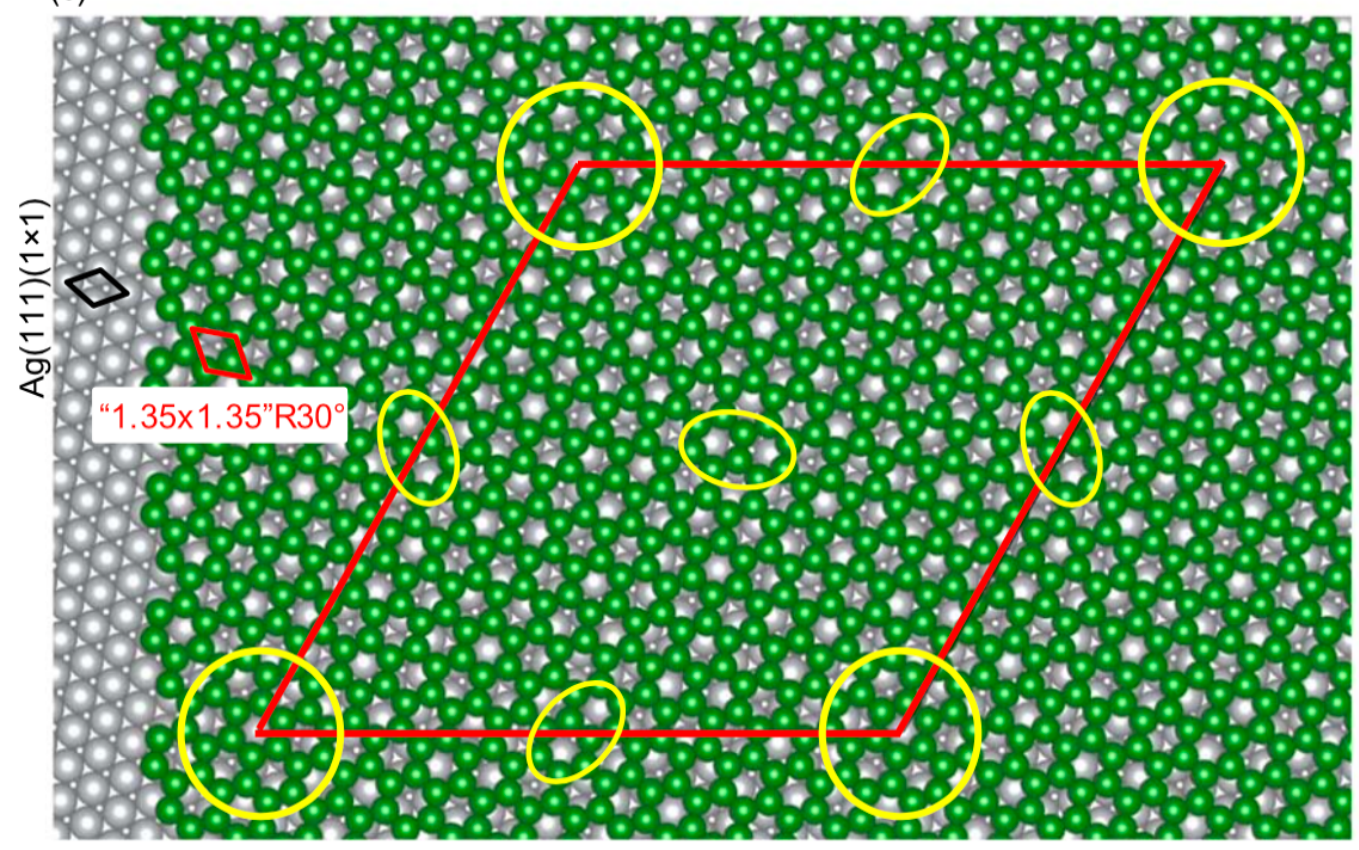

Fig. 3: Diffraction pattern and real space images of germanene obtained by segregation. (A) LEED pattern recorded at $63 \mathrm{eV}$. (B) STM image recorded in a constant current mode with tunneling current of $200 \mathrm{pA}$ and sample bias voltage of $+0.3 \mathrm{~V}$. The unit cell of " $1.35 \times$ $1.35)$ "R $30^{\circ}$ is indicated by the small lozenge. (C) Ball model of the germanene sheet on $\mathrm{Ag}(111)$, locally keeping a germanene unit cell of $(1.35 \times 1.35) \mathrm{R} 30^{\circ}$. The germanene $(3 \sqrt{2} 1 \times 3 \sqrt{2} 1) \mathrm{R} 10.9^{\circ}$ superstructure matches a $\mathrm{Ag}(111)(7 \sqrt{ } 7 \times 7 \sqrt{ } 7) \mathrm{R} 19.1^{\circ}$ supercell, indicated by the lozenge. The areal positions corresponding to the hexagon and line protrusions are marked by a solid yellow line.

By deposition of $\mathrm{Ge}$ onto $\mathrm{Ag}(111)$ surfaces kept at $150^{\circ} \mathrm{C}$ two distinct phases associated with low-buckled germanene have been observed by STM, LEED and angle-resolved photoemission spectroscopy (ARPES); ab initio calculations helped in their understanding. ${ }^{26}$ ) At lower coverage a first phase exhibits an atom-resolved tensile strained honeycomb lattice, partially commensurate with the substrate to form a striped phase (SP). At higher coverage, a second one consistent with a honeycomb lattice with a lattice constant incommensurate with the substrate but very close to the theoretical value for freestanding germanene, is a quasi- 
freestanding phase (QP). The SP can be driven into the QP by additional Ge deposition. Band mapping along with first- principles calculations with proposed atomic models for both phases reveal that the SP is subjected to significant band renormalization and develops an interface state due to its partial commensuration with the substrate. Instead, only the QP possesses the characteristic $\sigma$ band of freestanding germanene because the incommensurate structure leads to a weaker and smoothed interfacial interaction. These results indicate that $\mathrm{Ag}(111)$ provides a relatively gentle support for the formation and transformation of the dual germanene phases, similarly to the formation of silicene phases on the same support. They further illustrate how the competitive formation of $\mathrm{Ge}-\mathrm{Ge}$ covalent and $\mathrm{Ge}-\mathrm{Ag}$ interfacial bonds and adlayersubstrate commensurability play a key role in the electronic structure of the dual germanene phases.

Still upon deposition onto $\mathrm{Ag}(111)$, but at a slightly higher temperature of $177^{\circ} \mathrm{C}$, Du and coll., ${ }^{27)}$ fabricated thick germanium nanosheets, starting from an initial Ag2Ge surface alloy. ${ }^{28)}$ Strikingly, these silver films supported semiconducting nanosheets were terminated by a germanene monolayer dis- playing a $(\sqrt{3} \times \sqrt{3})$ superstructure. A linear-like energy-momentum dispersion, and a large Fermi velocity were derived from pronounced quasiparticle interference patterns, pointing to Dirac fermion characteristics. Furthermore, theoretical simula- tions revealed the opening of a sizeable band gap corresponding to RT, at the center $\Gamma$ of the SBZ because of the $(\sqrt{3} \times \sqrt{3})$ reconstruction. Clearly, the high charge carrier mobility, the sizable band gap, and the semiconducting character of the Ge (111) nanosheets with tunable thickness acting as a dielectric layer, may favour nanotechnology applications of this out- standing material. With the introduction of a thin h-AIN buffer layer the growth of 2D germanene sheets is preserved at a substrate temperature of $450{ }^{\circ} \mathrm{C}$, which is also promising for future applications. ${ }^{29)}$

Here, it is worth mentioning that multilayer germanene sheets harboring Dirac fermions have been grown in situ for the first time on $\mathrm{Au}(111)$ surfaces. ${ }^{30)}$

Concerning potential applications, it is worth mentioning here that besides the results obtained in situ under UHV reviewed in this article, wet chemical preparation methods yield highly promising Ge-based synthetic materials. ${ }^{9}$ ) This is the case of bulk germanane (van der Waals bonded stacks of fully hydrogenated germanene), which can be easily exfo- liated down to a monolayer, leading to a direct band gap opening at the $\Gamma$ point, ${ }^{31)}$ as well as functionalized germananes. ${ }^{32)}$ A fascinating aspect is that ultra-small germanene quantum dots, ${ }^{33)}$ like silicene nanosheets, ${ }^{34)}$ promise high clinical potential in nanomedicine.

\section{Stanene}

As silicene and germanene, stanene (rarely written as stannene, and sometimes called tinene) shows a quantum spin Hall effect due to the SOC: at the Dirac points fundamental band gaps open up, which can be tuned by applying a perpendicular electric field, inducing a topological phase transition. ${ }^{35,36)}$ The gap is $73 \mathrm{meV}$ in stanene compared to $23 \mathrm{meV}, 1.55$ in germanene and silicene, but negligible (only $8 \mu \mathrm{eV}$ ) in graphene. This means that stanene can be a $2 \mathrm{D}$ topological insulator above RT, while germanene can be slightly below.

In 2015, Zhu et al. reported the first synthesis of single- layer stanene upon growth by MBE on a metallic $\mathrm{Bi}_{2} \mathrm{Te}_{3}(111)$ surface; it presented no signature of a topolo- gical state. ${ }^{37)}$ Due to 
compressive strain, it was strongly buckled, the growth fashion of tin on this surface actual following an islands mode.

At variance, a nearly planar stanene structure in large domains was realized by Yuhara et al. on a $\mathrm{Ag}(111)$ surface after growth of an initial $\mathrm{Ag}_{2} \mathrm{Sn}$ surface alloy, acting as a template, where the growth fashion is indicated in Fig. 4 (a). ${ }^{20}$ ) To the best of our knowledge, this is the first evidence of a 2D elemental Xene generated in such a way. The crystalline structure was ascertained synergetically by STM, high-resolution synchrotron radiation photoemission valence band and core-level spectroscopies, and advanced first- principles calculations. As displayed in Figs. 4(b) and 4(c), the STM images show that stanene forms a nearly planar structure in large domains. A detailed core-level spectroscopy analysis as well as DFT calculations [see Fig. 4(d)] revealed that the stanene sheet overlays an ordered Ag2Sn 2D surface alloy, not directly residing on a bulk-terminated $\mathrm{Ag}(111)$ surface. However, the electronic structure measured in ARPES does not reveal a non-trivial behavior; instead, it exhibits a characteristic 2D band with parabolic dispersion due to the non-negligible interaction with the underlying surface alloy.

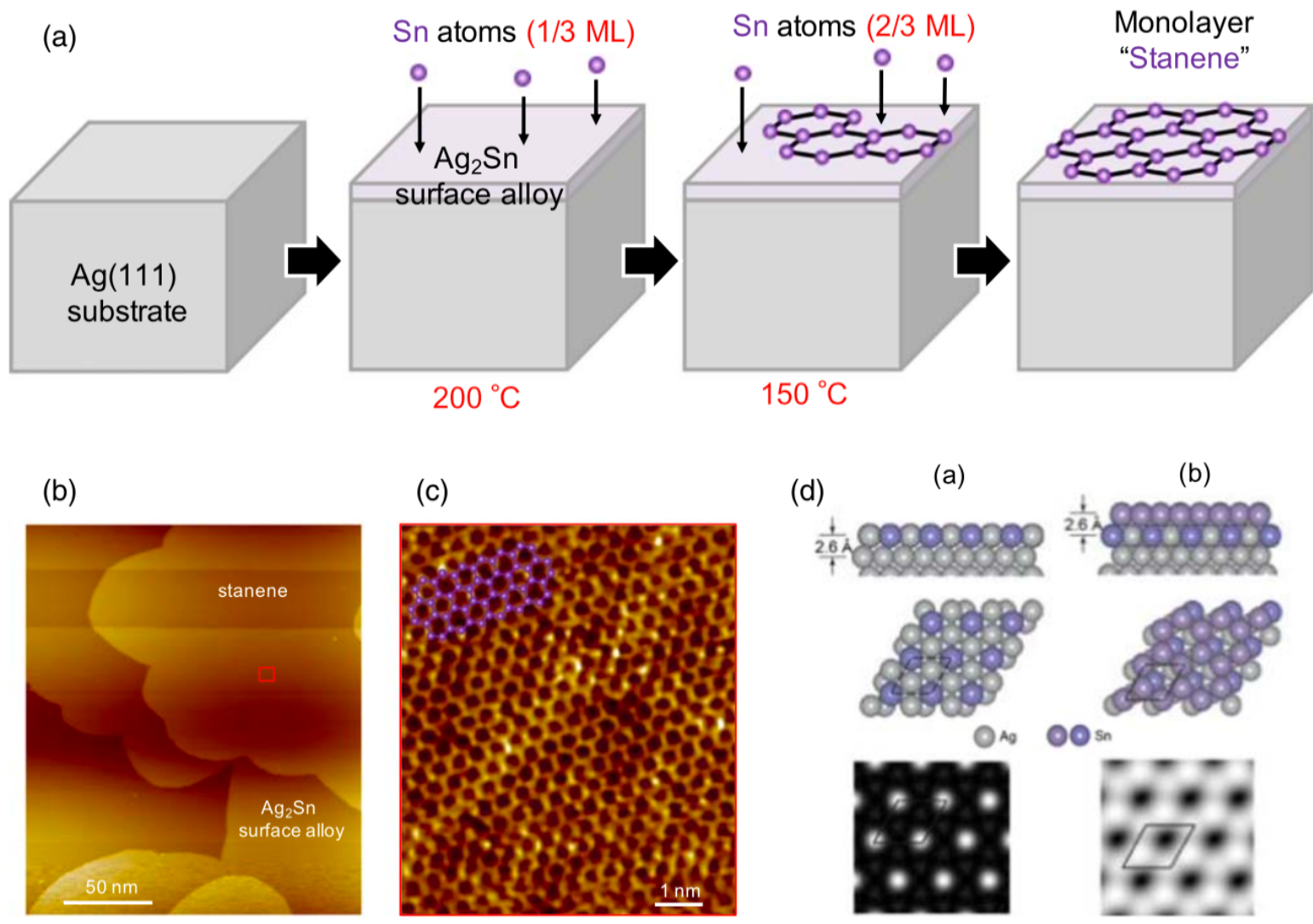

Fig. 4: Synthesis of nearly planar stanene on $\mathrm{Ag}(111)$. (A) Scheme of the growth fashion. (B) Large scale STM image showing stanene and a partly uncovered zone of the Ag2Sn template. (C) Honeycomb aspect of the stanene layer in STM imaging; inset: ball model of its atomic geometry. (D) Structural models (side and top views) and their simulated STM images of (a) the Ag2Sn surface alloy and (b) stanene on the Ag2Sn surface alloy.

Soon after this first realization of nearly flat stanene, 2D high-quality ultraflat stanene has been fabricated on $\mathrm{Cu}(111)$ by low-temperature $\mathrm{MBE}^{38}$ ) Due to the epitaxial effect of the substrate, its lattice constant is as high as $0.51 \mathrm{~nm}$ and it shows an in-plane s-p band inversion together 
with a SOC- induced topological gap at the $\Gamma$ point of $\sim 0.3 \mathrm{eV}$, far exceeding the RT thermal fluctuation energy. Hence, this ultraflat monolayer stanene displays clear non-trivial features in ARPES experiments, while topologically derived boundary states were observed by STM measurements. As superconductivity in few-layer stanene has been recently discovered, ${ }^{39)}$ further exploration of 2D superconductivity or even Majorana fermions could be envisaged. Furthermore, first-principles calculations predicted that ultraflat plumbene could also be fabricated on $\mathrm{Cu}(111)$, with also an $\mathrm{s}-\mathrm{p}$ band inversion together with a SOC-induced gap at the $\Gamma$ point. ${ }^{38)}$ All these striking findings open tantalizing prospects for further exploring $2 D$ topological physics and device applications.

Stanene was also grown on other substrates, with promises of many exciting properties. We refer to Ref. 9, and especially to the most recent review by Lyu et al. for new electronic and spintronic applications. ${ }^{40)}$

\section{Plumbene}

Beginning of January 2020 , a search in the Web of Science with plumbene in topic yields only 10 results, the first paper having been published only in 2016. Nine papers are based on theoretical calculations; just the most recent one in July 2019 by Yuhara et al. reports the first experimental realiza- tion of plumbene. ${ }^{1)}$ This reflects the initial skepticism of the $2 \mathrm{D}$ material community about the possibility to synthesize the heaviest cousin of graphene with the highest intrinsic SOC.

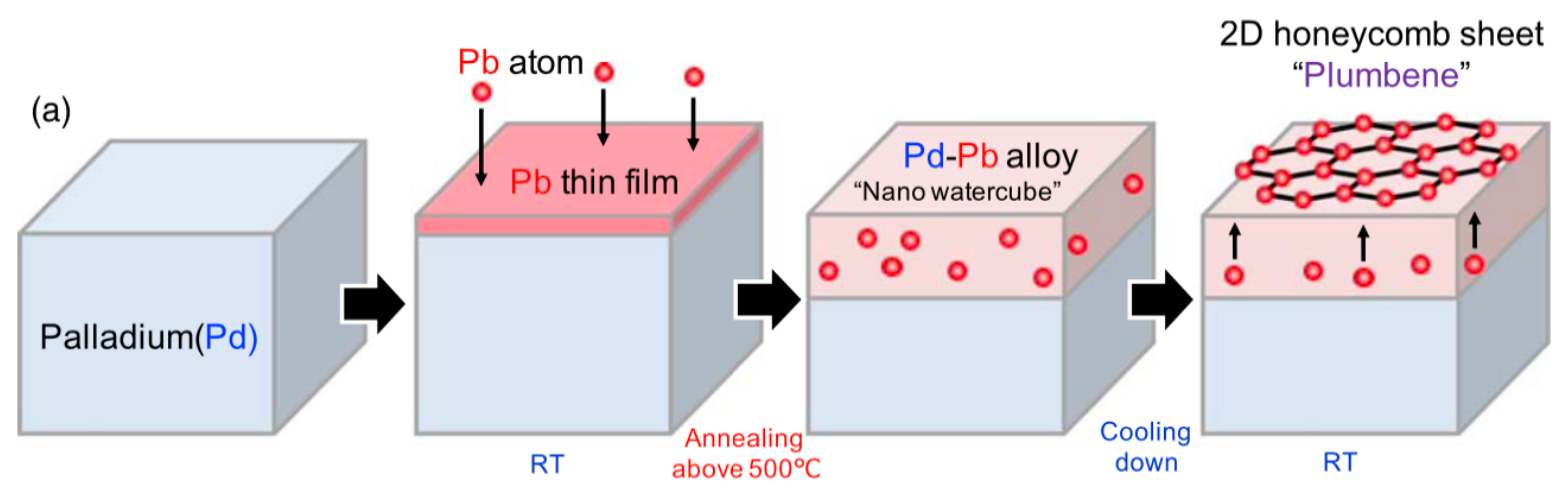

(b)

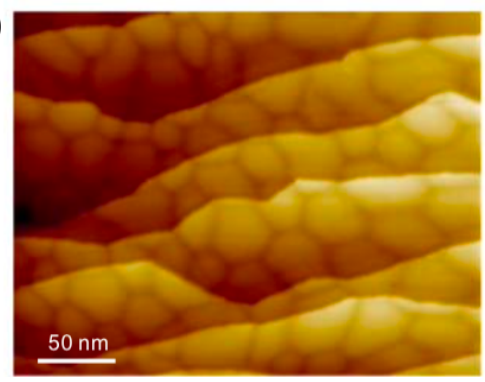

(c)

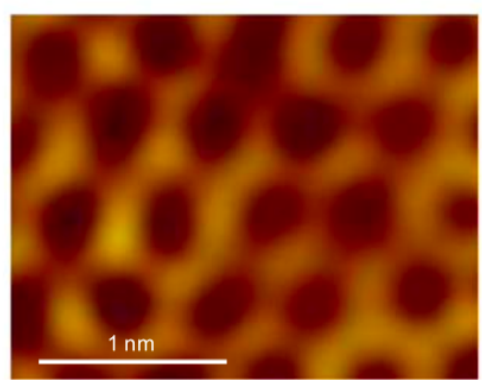

Fig. 5: First experimental realization of plumbene on a "Nano WaterCube". (A) Scheme of the growth method. (B) Large scale STM image showing the bubblelike aspect of the $\mathrm{Pd}(1-\mathrm{x}) \mathrm{Pb}$ dilute alloy thin film-coined a "Nano WaterCube"-because of its resemblance with the facade of the swimming pool (nick- name, the "Water Cube") of the 2008 Olympics in Beijing. (C) Honeycomb structure of the plumbene overlayer in STM imaging. 
However, with the smart choice of a palladium single crystal substrate, Yuhara and co-workers at Nagoya University in Japan could provide compelling evidence of the epitaxial growth of large-area plumbene sheets. The synthesis has been achieved on the (111) surface of a $P d(1-x) P b x$ dilute alloy thin film, using a segregation method from the $\mathrm{Pd}(111)$ substrate, as depicted in Fig. 5(a).

This dilute alloy thin film exhibits in STM imaging a unique surface morphology resembling the famous Weaire- Phelan bubble structure of the Olympic "WaterCube" in Beijing. ${ }^{41)}$ The "soap bubbles" of this "Nano Water Cube" are adjustable with their average sizes (in-between 15 and $80 \mathrm{~nm}$ ) related to the $\mathrm{Pb}$ concentration $(\mathrm{x}<0.2)$ dependence of the lattice parameter of the $\mathrm{Pd}_{(1-\mathrm{x})} \mathrm{Pb}_{\mathrm{x}}(111)$ alloy surface. Angle-resolved core-level spectroscopy measurements demonstrate that a lead sheet overlays the $\mathrm{Pd}(1-\mathrm{x}) \mathrm{Pb}_{\mathrm{x}}(111)$ alloy. The atomic-scale STM images of this $\mathrm{Pb}$ sheet show a planar honeycomb structure with a unit cell in accordance to that of freestanding plumbene.

High band gaps are predicted in decorated plumbene, which could lead to a quantum spin hall insulator behavior and fascinating high-temperature applications. ${ }^{42)}$ Indeed, synthesizing such sheets while keeping their non-trivial characteristics remains a non-easy challenge.

\section{Summary and outlook}

All the Group 14 2D honeycomb materials beyond graphene, the lineage from silicene, first realized in 2012, to plumbene, realized in 2019, have been now synthesized. The virtual possibilities opened by Takeda and Shiraishi in 1994, 10 years before the advent of graphene, have now materialized. This puts an end to a long Holy Grail quest, but opens tantalizing prospects for the practical implementation of non- trivial emergent phenomena in nanoelectronics, and nano- photonics, in spintronics, and, last but not least, in quantum computing with the search for Majorana fermions.

These novel 2D materials share the optimum geometry, which has intrigued mankind for millennia, that is, the honeycomb array structure constructed by bees upon mas- sively parallel working under natural collective intelligence. Noteworthy, is that it is only in 2001 that the socalled classical honeycomb conjecture, namely, the intuition that the best way to partition a surface into regions of equal areas with the smallest total perimeter, ${ }^{12}$ ) has been mathematically solved by Thomas Hales. ${ }^{42}$ )

Strikingly, the realization of plumbene relies also on a template, a thin $\mathrm{Pd}(1-\mathrm{x}) \mathrm{Pb}_{\mathbf{x}}$ dilute alloy, ${ }^{1)}$ reminiscent of the special 3D foam geometry derived in 1994 by Weaire and Phelan, who found a counter-example to the famous Kelvin's conjecture that a b.c.c. arrangement of minimal tetrakaideca- hedron divides space into equal cells of minimum surface area. ${ }^{41}$ ) This conjecture, which had stood for over one hundred years, has thus been ruined, but the mathematical problem remains entirely open.

Here, nanoarchitectonics meets mathematics and real archi- tecture: the Weaire-Phelan construction has inspired the 2008 Olympics "Water Cube" in Beijing, while, conversely, the soccer ball atomic geometry of the $\mathrm{C}_{60}$ fullerene, has been inferred from the geodesic dome of the 1967 Montreal Expo, designed by famous architect Buckminster Füller. 
Acknowledgments

The authors are grateful to Nagoya University Synchrotron Radiation Research Center for financial support for CLS measurements, which were conducted at the BL7U of Aichi Synchrotron Radiation Center, Aichi Science \& Technology Foundation, Aichi, Japan (No. 2018001). J.Y. acknowledges financial support from the Murata Science Foundation in 2019. G.L.L. acknowledges support from Nagoya University thanks to an "Eminent Foreign Scientist" Invitation Award in 2015/ 2016, and a "Principle Investigator" Award in 2019/2020, as well as an Invitational Fellowship for Research in Japan by the Japan Society for the Promotion of Science (JSPS) in 2017.

\section{References}

1) J. Yuhara, B. He, N. Matsunami, M. Nakatake, and G. Le Lay, Adv. Mater. 31, 1901017 (2019).

2) P. Vogt, P. De Padova, C. Quaresima, J. Avila, E. Frantzeskakis, M. C. Asensio, A. Resta,

B. Ealet, and G. Le Lay, Phys. Rev. Lett. 108, 155501 (2012).

3) A. Fleurence, R. Friedlein, T. Ozaki, H. Kawai, Y. Wang, and Y. Yamada-

Takamura, Phys. Rev. Lett. 108, 245501 (2012).

4) C.-L. Lin, R. Arafune, K. Kawahara, N. Tsukahara, E. Minamitani, Y. Kim,

N. Takagi, and M. Kawai, Appl. Phys. Express 5, 045802 (2012).

5) K. Takeda and K. Shiraishi, Phys. Rev. B 50, 14916 (1994).

6) S. Cahangirov, M. Topsakal, E. Aktürk, H. Şahin, and S. Ciraci, Phys. Rev. Lett. 102, 236804 (2009).

7) A. J. Mannix et al., Science 350, 1513 (2015).

8) F. Reis, G. Li, L. Dudy, M. Bauernfeind, S. Glass, W. Hanke, R. Thomale,

J. Schäfer, and R. Claessen, Science 357, 287 (2017).

9) P. Vishnoi, K. Pramoda, and C. N. R. Rao, ChemNanoMat 5, 1062 (2019).

10) S. Cahangirov, S. Hasan, G. Le Lay, and A. Rubio, Introduction to the Physics of Silicene and Other 2D Materials. Lecture Notes in Physics (Springer, Berlin, 2017).

11) P. Vogt and G. Le Lay (ed.) Silicene Prediction, Synthesis, Application, NanoScience and Technology Book (Springer, Berlin, 2018).

12) N. Takagi, C. L. Lin, and R. Arafune, in Silicene Structure, Properties and Applications, ed. M. Spencer and T. Morishita, (Springer, Berlin, 2016), p. 143.

13) G. L. Lay, E. Salomon, and T. Angot, in Silicene, Germanene, and Stanene in 2D Materials Properties and Devices, ed. P. Avouris et al. (Cambridge University Press, Cambridge, 2017), p. 458.

14) M. E. Dávila, G. Le Lay, and J. Cerdá, in 2D Semiconductor Materials and Devices, ed. D. Chi et al. (Elsevier, Amsterdam, 2020), p. 221.

15) Y. Zhang, A. Rubio, and G. L. Lay, J. Phys. D: Appl. Phys. 50, 053004 (2017).

16) M. Ezawa, E. Salomon, P. De Padova, D. Solonenko, P. Vogt, M. E. Dávila, A. Molle, T. Angot, and G. Le Lay, La Rivista del Nuovo Cimento 41, 175 (2018).

17) (2014), Research Fronts 2014: 100 Top-Ranked Specialties in the Sciences and Social Sciences; The National Science Library, Chinese Academy of Emerging Technology Analysis.

18) Research Fronts 2019 [https://discover.clarivate.com/

ResearchFronts2019_EN] (accessed March 3, 2020).

19) I. Matsuda et al., Phys. Rev. B 68, 085407 (2003).

20) J. Yuhara, Y. Fujii, K. Nishino, N. Isobe, M. Nakatake, L. Xian, A. Rubio, and G. Le Lay, 2D Mater. 5, 025002 (2018).

21) M. E. Dávila, L. Xian, S. Cahangirov, A. Rubio, and G. Le Lay, New J. 
Phys. 16, 095002 (2014).

22) G. Le Lay et al., Surf. Sci. 307-309, 280 (1994).

23) M. Derivaz, D. Dentel, R. Stephan, M.-C. Hanf, A. Mehdaoui, P. Sonnet, and C. Pirri, Nano Lett. 15, 2510 (2015).

24) D. A. Muzychenko, S. I. Oreshkin, V. I. Panov, C. Van Haesendonck, and

A. I. Oreshkin, Nano Res. 12, 2988 (2019).

25) D. Sciacca, N. Peric, M. Berthe, L. Biadala, C. Pirri, M. Derivaz,

N. Massara, P. Diener, and B. Grandidier, J. Phys.: Condens. Matter 32, 055002 (2019).

26) C.-H. Lin et al., Phys. Rev. Mater. 2, 024003 (2018).

27) J. Zhuang et al., Adv. Sci. 5, 1800207 (2018).

28) J. Dalmas, H. Oughaddou, C. Léandri, J.-M. Gay, G. Le Lay, G. Tréglia,

B. Aufray, O. Bunk, and R. L. Johnson, Phys. Rev. B 72, 155424 (2005).

29) F. d'Acapito, S. Torrengo, E. Xenogiannopoulou, P. Tsipas, J. M. Velasco, D. Tsoutsou, and A. Dimoulas, J. Phys.: Condens. Matter 28, 045002 (2016).

30) M. E. Dávila and G. Le Lay, Sci. Rep. 6, 20714 (2016).

31) E. Bianco, S. Butler, S. Jiang, O. D. Restrepo, W. Windl, and J. E. Goldberger, ACS Nano 7, 4414 (2013).

32) J. Sturala, J. Luxa, S. Matějková, Z. Sofer, and M. Pumera, Nanoscale 11, 19327 (2019).

33) J. Ouyang et al., Angew. Chem. Int. Ed. 58, 13405 (2019).

34) H. Lin, W. Qiu, J. Liu, L. Yu, S. Gao, H. Yao, Y. Chen, and J. Shi, Adv.

Mater. 31, 1903013 (2019).

35) L. Matthes, O. Pulci, and F. Bechstedt, J. Phys.: Condens. Matter 25, 395305 (2013).

36) M. Ezawa, Appl. Phys. Lett. 102, 172103 (2013).

37) F. Zhu, W. Chen, Y. Xu, C. Gao, D. Guan, C. Liu, D. Qian, S.-C. Zhang, and J. Jia, Nat. Mater. 14, 1020 (2015).

38) J. Deng et al., Nat. Mater. 17, 1081 (2018).

39) M. Liao et al., Nat. Phys. 14, 344 (2018).

40) J.-K. Lyu, S.-F. Zhang, C.-W. Zhang, and P.-J. Wang, Ann. Phys. 531, 1900017 (2019).

41) D. Weaire and R. Phelan, Philos. Mag. Lett. 69, 107 (1994).

42) T. C. Hales, Discrete Comput. Geom. 25, 1 (2001). 